\title{
La nueva gerencia pública en el Perú
}

\author{
The new public management in Peru
}

\section{RESUMEN}

Objetivo: Este artículo responde a la necesidad de comprender las nuevas corrientes en la conducción de las organizaciones públicas peruanas, así como la influencia que ejerce el talento humano en los directivos modernos de la administración pública. Método: El artículo es de alcance descriptivo y método inductivo, pues si bien localiza la población-muestra de estudio en los funcionarios gerenciales de la organización local (Municipalidad Distrital de San Isidro, en Lima-Perú), las características comunes y/o similares de las organizaciones públicas en el país, así como los elementos cognitivos del talento humano, la califican como de alcance general y a todos los niveles de la organización pública. Resultado: La Gerencia pública es el resultado moderno y evolutivo en la conducción de las organizaciones públicas, resultante de una progresiva adaptación de la legislación del servicio civil a la cambiante realidad que incorpora a la tradicional gestión del Estado, métodos y prácticas reservadas en antaño para la empresa privada. Palabras claves: Gestión municipal; talento humano; conocimientos directivos; habilidades gerenciales; actitud organizacional; liderazgo; destreza funcional; visión y misión gerencial.

\begin{abstract}
Objective: This article responds to the need to understand the influence of human talent in the management and development of public managers, in its dimensions: components of knowledge, skills and attitudes (know how to be, know know, know how to do). In addition, it is part of the research of the public sector, by exposing the progress of the study of the new management of human talent in Peruvian public organizations. Method: The investigation is of descriptive scope and inductive method, because although it locates the population-sample of study in the managerial officers of the local organization (District Municipality of San Isidro, in Lima-Peru), the common characteristics and / or similar of public organizations in the country, as well as the cognitive elements of human talent. Result: A modern and evolving term of the administration of the personnel that works in the State has been established and has been observed through the modifi-
\end{abstract}

\section{Luis Alberto Arias Mercado}

Lariasm@unmsm.edu.pe

Universidad Nacional Mayor de San Marcos, Facultad de Ciencias Administrativas. Lima, Perú 
cation in the Civil Service Legislation in recent years. In addition, contributions can improve their adaptation and / or adaptation to the conditions of public agencies at the central and regional levels of the country.

Keywords: Municipal management; human talent; management skills; managerial skills; organizational attitude; leadership; functional skills; vision and managerial mission.

\section{INTRODUCCIÓN}

Desde los inicios de la Globalización mundial en los 80, se viene replanteando el papel del Estado en la sociedad a través de las políticas públicas, tanto en su formulación como en su implementación. Esto direcciona la atención hacia el histórico paradigma Weberiano y su modelo de gestión burocrático/jerárquico, que en los últimos 40 años tuvo mayor notoriedad en los variados procesos de reforma del Estado.

Sin embargo, en opinión de Blutman \& Felcman, (2011) dicho modelo organizacional de gestión pública se está resquebrajando, en el sentido de que "las firmes creencias sobre el Estado mínimo, la desregulación y la infalibilidad de la mano invisible del mercado" empiezan a crear dudas en lo que podría llevar a una ruptura paradigmática.

Entonces, surge como tema de reflexión académica los modelos organizacionales de gestión más apropiados para transformar el Estado y las instituciones públicas haciéndolas adecuadas para la satisfacción de necesidades e intereses sociales que se dirigen a su bienestar y desarrollo.

En esta inevitable vinculación entre la sociedad y el Estado, en el marco de los cambios en las relaciones internacionales de los países en el presente siglo XXI se observa que la primacía de las concepciones neoliberales trae los resultados siguientes: "el desarrollo desigual de las distintas regiones del mundo, la segmentación del mundo en bloques económicos en competencia..., el surgimiento de nuevos valores culturales, económicos, políticos, sociales y ecológicos, la marginación de los países más pobres y de menor desarrollo tecnológico y, entre otros, el desenvolvimiento sin restricciones de las leyes del mercado. (Alvarez, 2012).

Ante este contexto histórico y factual de la sociedad en su relación con el Estado con una mirada epistemológica de la ciencia de la Administración se puede afirmar que, como menciona Longo (2002) en aquellos sistemas estatales "encontramos -como ocurre en las democracias avanzadas- los elementos básicos constitutivos de una administración profesional, la acción pública ha venido estando repartida durante décadas, con arreglo al conocido modelo weberiano, entre dos grandes protagonistas: una clase política, investida de autoridad a través de los mecanismos de la democracia representativa, y una función pública profesional, regida por el sistema de mérito" (pág. 1), esto se apoya al nuevo concepto de gerencia social y pública.

Asociada a este contexto global del Estado y la gestión pública se aprecia una tendencia de la nueva realidad mundial calificada como la era del conocimiento denominada la guerra por el talento que se caracteriza porque desde "las empresas se demandan personas talentosas como ventaja competitiva. Contar con mejor talento tiene una inmensa importancia donde los talentos escasean, cada vez las personas son volátiles y asumen compromisos a corto plazo, pero al mismo tiempo las personas exigen mucho más". (Michaels, Handfield, \& Beth A., 2003)

El segundo nivel del contexto del artículo para construir el objeto de estudio, se refiere al sistema organizacional del sector público del país, donde se observa que el Estado peruano tiene una sensible disminución de su estructura y rol, así como el riesgo de estancamiento del desarrollo económico sostenido que ha mantenido en la última década, donde el crecimiento superior al 7\% anual, lo posicionó como uno de los países líderes de América Latina y del mundo.

El relativo exitoso manejo económico sumado a la proliferación de manifestaciones sociales en contra de la actividad extractiva de un lado y de otro, la falta de solidez y confianza en las instituciones tutelares (Poder Ejecutivo, Congreso, Poder Judicial , Universidades, etc.), a lo que añade la inusitada elevación de la delin- 
cuencia e inseguridad ciudadana con la aparición de novedosas modalidades delictivas (sicariato, extorsión, secuestro, etc.), configuran un marco que desalienta la inversión extranjera y nacional conllevando a un panorama poco prometedor para el ciudadano de a pie.

Esta situación de riesgo, acompañada del agitado sistema político pone a prueba la fortaleza de los organismos públicos y especialmente a la pericia y gestión de las personas que las conducen, considerando que el Estado en todos sus niveles (nacional, regional y local) es responsable en gran medida de los hechos descritos, y a la vez, tienen la enorme responsabilidad de contribuir al rescate y recuperación de los espacios perdidos.

Esta situación organizacional del Estado se asocia con el contexto administrativo en el país Chesman, (2010) donde el gobierno en la última década inició el proceso de cambio en la administración pública mediante la Ley Marco de Modernización de la Gestión Pública (30 de Enero de 2002) con la finalidad de mejorar la gestión pública y construir un Estado democrático, descentralizado y al servicio de la ciudadanía.

Posteriormente se promulgó el D.S. $\mathrm{N}^{\circ} 004$ 2013-PCM que aprobó la Política nacional de Modernización de la gestión pública, con el objetivo de orientar a las entidades públicas hacia una gestión orientada a resultados al servicio del ciudadano, cuyos avances más importantes son el proceso de descentralización, la simplificación administrativa y la transparencia de un lado, y de otro, la reforma del servicio civil con el sistema "servir" como respuesta a que en el siglo pasado no se logró una reforma integral de gestión a nivel operacional que pueda afrontar la debilidad estructural del aparato estatal y la falta de organización sistémica.

La Gestión de personas es uno de los componentes del proceso de modernización de la gestión pública que tiene como propósitos: profesionalizar la función pública, contar con funcionarios y servidores idóneos para el puesto que desempeñan, mantenerlos motivados para servir y para que cumplen con los principios éticos de la función pública.

En los procesos de modernización y reforma de la administración pública, tienen rol protagónico las personas que planifican, guían y toman las decisiones en los cargos directivos y gerenciales, cuyo desempeño tiene alta gravitación en el éxito de la gestión pública local.

Ellos son los encargados de interpretar las aspiraciones ciudadanas, convertirlas en planes, programas y proyectos coherentes y viables, con la asignación de recursos y en un marco de condiciones materiales, espirituales y emocionales cuyo equilibrio no debe ser excusa para dejar de ser eficiente y satisfactoria. Su dirección hacia el mejoramiento de las condiciones actuales de la organización y gestión del futuro deseado, será el producto tangible de este desempeño.

Así la Gerencia Pública, es el nuevo término acuñado para identificar y personificar al tradicional jefe de una dependencia pública hasta el tercer nivel organizacional y que identifica a los protagonistas de la gestión con la base teórica y científica de la Administración Pública que ha sido objeto de estudios en sus múltiples dimensiones de su desempeño técnico y profesional, así como el rendimiento organizacional en concordancia a la visión de desarrollo nacional.

En estas condiciones los gerentes públicos, en su condición de líderes gestores de las organizaciones tutelares, deben convertirse en las piezas claves de la recuperación económica y social de los distintos espacios y niveles de la vida ciudadana. Su efectividad y tino para tomar las decisiones acertadas y conducir hacia los caminos de crecimiento y desarrollo, precisarán de cualidades más allá de la simple pericia técnica o profesional. Su talento humano determinará el nivel de éxito en su empeño.

Al respecto, cabe señalar que uno de los principales signos de deterioro de la administración del Estado Peruano no se grafica en la ausencia de recursos sino en la deficiente ejecución del gasto público. Paradójicamente en circunstancias de mayores necesidades y urgencias para la población, los niveles de gasto no alcanzan los estándares apropiados tanto en la cantidad de recursos destinados, como en la calidad del gasto, produciéndose dispendio en proyectos no productivos o de segunda prioridad, no vinculados con el crecimiento y como destino hacia los sectores de mayor necesidad. 
Los controles públicos se debilitan y los niveles de corrupción alcanzan niveles impresionantes.

Como pocas veces se ha visto, en la última década se han procesado y condenado a ex presidentes y numerosos gobernadores regionales y alcaldes por delitos de corrupción, entre otros delitos asociados a la creciente criminalidad, conllevando al país a una coyuntura problemática con signos de crisis económica y moral, que requiere con carácter de urgente fortalecer la gestión pública desde el nivel local y revisar la influencia de la gestión de los gerentes públicos en dicho empeño.

Ello significa elevar los estándares de efectividad en la gestión pública, mediante indicadores de mejoramiento del uso de los recursos públicos (eficiencia) y obtener los resultados esperados, en el tiempo preciso (eficacia), para que se conviertan en factores determinantes de los proyectos estratégicos de recuperación, crecimiento e impulso al desarrollo de las condiciones de vida ciudadana.

La justificación metodológica y de carácter fáctica del artículo se orienta hacia la búsqueda de alternativas académicas y profesionales que impulse a nuevos niveles de calidad y excelencia de los servicios públicos, ya que a la fecha existe una fuerte carencia de resultados favorables con baja productividad en la atención de las demandas ciudadanas a cargo de las entidades del Estado y particularmente de los gobiernos locales. Se requiere inyectar a las organizaciones públicas del Perú, una renovada fuerza que impacte positivamente en los resultados en favor de la ciudadanía, mediante la gestión de las personas basada en el talento humano y el desarrollo del gerente público con el alineamiento hacia la visión, misión y objetivos públicos.

Los componentes y tipos de talento que influyen en las áreas del desarrollo del gerente público constituyen un factor de aplicación dentro de la corriente de la modernización de la gestión pública qué beneficiará a los ciudadanos mediante la calidad de la prestación de los servicios públicos en las municipalidades cumpliendo así con la representación de los intereses de los vecinos en el territorio local.

El aporte del artículo, considerando los elementos comunes de las organizaciones pú- blicas (sistemas administrativos y de gestión) y los componentes cognitivos del talento humano, abona en la construcción de modelos aplicables, adaptables y de adecuación a las singulares condiciones de otros organismos públicos de nivel nacional y regional. La composición afín y/o similar de las organizaciones públicas, los resultados de la presente investigación resultan perfectamente ajustables y/o adaptables a los gerentes públicos de organizaciones del Estado de distinto nivel (nacional y regional) y naturaleza (empresas públicas, organismos descentralizados, entre otras).

\section{MATERIAL Y MÉTODO}

El artículo se encuentra diseñada a partir de las reflexiones teóricas y fácticas de la administración pública local, además focaliza su atención en la figura icónica del gerente público, en su rol protagónico de prestatario de bienes y servicios a la ciudadanía y principal responsable del éxito (o fracaso) de la gestión institucional.

En ese sentido, sobre el gerente público recae la interrogante metodológica de entender cuánto influye su gestión y desarrollo en la dirección del talento humano de los demás servidores públicos.

La información y concepción de la temática estudiada se obtuvo mediante la observación directa, así como la lectura de fuentes primarias, encuestas a la población y entrevistas a los principales gestores municipales.

\section{RESULTADOS}

Es evidente la adopción de una nueva corriente administrativa en el Estado vinculada a la Nueva Gerencia Pública. El talento humano aparece en este contexto como una determinante influencia en la gestión y desarrollo del gerente público.

El tradicional consentimiento de la condición técnica, experta y/o profesional para ocupar un cargo directivo/gerencial en la administración pública local, ha dejado de ser suficiente como garantía de niveles óptimos de eficiencia, eficacia, economía, oportunidad y resultados esperados por la ciudadanía.

Se precisa que el gerente público, además de poseer principios y solvencia ética y moral, debe ser talentoso, en su significado dimensio- 
nal de conocimientos (saberes), habilidades (saber hacer) y actitudes (querer hacer).

Esta trilogía del talento humano, es exigente para garantizar un rendimiento eficiente y honesto en el manejo de los recursos públicos, eficaz para la obtención de resultados en la forma y tiempos programados y efectivo en la satisfacción de las expectativas y demandas ciudadanas.

Podrán emprenderse políticas y programas estratégicos de promoción y desarrollo de la gerencia pública, como principales activadores de la modernización, innovación y resultados de alto impacto en las organizaciones del Estado.

\section{DISCUSIÓN}

Respaldamos la definición que ha dado la Carta Iberoamericana (CLAD, 2003) sobre la Función Pública que lo cataloga como un conjunto de coordinaciones institucionales (normas, estructuras, pautas culturales, políticas, procesos, prácticas) a través de los cuales se gestiona el empleo público con el fin de garantizar una óptima conducción de los RRHH y una Administración pública profesional y eficaz. Además, tal como lo dice Pagani. (2015) es un instrumento indispensable para la consecución de un mejor Estado que involucre todos los estamentos poder garantizar la calidad en el servicio público.

Idalberto Chiavenato (2011) afirma que "para movilizar y utilizar con plenitud a las personas en sus actividades, las organizaciones están cambiando los conceptos y modificando las prácticas gerenciales". Por ende, se manifiesta que las personas, en este caso los gerentes públicos, constituyen el elemento básico del éxito empresarial. Es así que "la estrategia constituye el plan general que la organización adopta para asegurarse de que las personas puedan cumplir la misión organizacional de manera adecuada."

Esta exploración pragmática de las novedades de gestión empresarial está conduciendo a las modernas organizaciones de regreso a lo fundamental: La energía humana mueve la Empresa, por lo tanto, Castillo (2018) menciona que "su éxito o fracaso depende de las ideas, actitudes, capacidades y motivaciones de sus integrantes“. La diferencia está en la gestión que se haga para captar y desarrollar el Talento Humano de las personas.

En el mismo sentido, el concepto enfocado a la Gestión del Talento Humano en el Sector Público se ha iniciado con el proceso de captación de personal para los puestos de gerentes, con factores modernos y diferenciadores.

El énfasis en las condiciones del talento humano, por sobre elementos tradicionales como los años de experiencia o la sobre calificación académica se convierte en el factor clave del éxito de toda organización, citado en Gálvez, (2016) de Ponce, 2001 "La administración del talento humano como factor clave de éxito en la gestión empresarial."

El talento humano "En el sentido más general, es la suma de las habilidades de una persona: sus dotes innatas, sus destrezas, conocimientos, experiencia, inteligencia, juicio, actitud, carácter e iniciativa. Comprende también su capacidad de aprender y desarrollarse" Ed, Michaels, (2002)

Vallejos (2015) menciona la existencia de tres aspectos esenciales en el talento de las personas "que reflejan la competencia individual. 1. Conocimiento (es el saber) 2. Habilidad. (es saber hacer) y 3. Actitud (es como hacer)"

La existencia de una Administración Pública cada vez más eficiente, eficaz y protectora de los recursos; es "capaz de mejorar la calidad del servicio público, de permitir al sector público adaptarse de manera flexible y desde una óptica más estratégica a los cambios externos, y de favorecer la mejora de los resultados económicos nacionales" (Ibíd: 37).

Por otro lado, el CLAD (1998) formula un modelo, en un artículo llamado "Una Nueva Gestión Pública para América Latina”, que tiene en consideración la actividad empresarial del estado, por ende, "el gobierno no puede ser una empresa, pero sí puede tornarse más empresarial" (Ibíd: 4)

Por último, se puede afirmar que "un Gerente Público es un profesional altamente calificado, que es empleado por el Estado y está íntimamente involucrado en lo que se ha llamado: La profesión gubernamental". Es preciso recalcar la importancia de un Gerente Público 
quien es un funcionario de alto rango que es capaz de garantizar, mediante su liderazgo; la activa cooperación, participación y dedicación de su personal. En otras palabras, dicho por Duque (2006), "el Gerente Público es capaz, a través de su actuación, alcanzar altos niveles de eficiencia y productividad por parte del funcionamiento del Estado".

\section{CONCLUSIONES.}

\section{Conclusión General:}

La Nueva Gerencia Pública tiene en el Talento Humano una determinante influencia en el éxito de su gestión y desarrollo en beneficio de la comunidad a la que sirve.

\section{Conclusión Específica 1:}

El grado de conocimientos generales y específicos del gerente público, vinculados a las materias y disciplinas que se relacionan y contribuyen en forma directa e indirecta con la administración pública (economía, sociología, sicología, derecho, administración, etc. etc.), son influyentes en el desarrollo de su gestión y éxito profesional e institucional.

\section{Conclusión Especifica 2:}

La habilidad gerencial expresada en la destreza, pericia y acierto (entre otros elementos cognitivos) para la atención y solución eficiente, eficaz y oportuna de las demandas de la organización y sus clientes (usuarios/ciudadanos/vecinos), es un elemento concurrente al conocimiento gerencial y se expresa en la equilibrada y armónica combinación de las reglas públicas (políticas, estrategias, normas legales y similares) y la condición o capacidad discrecional que posee el gerente público.

\section{Conclusión Especifica 3:}

El Talento Humano en su dimensión de actitudes gerenciales, resulta influyente en la aplicación de los conocimientos y habilidades del gerente, así como en la toma de decisiones para la atención y solución de la pluralidad de asuntos (simples y complejos) de su gestión y desarrollo.
La actitud gerencial está relacionada con la activación de la voluntad o disposición del gerente para hacer (o dejar de hacer) y comportarse ante lo que le corresponde y/o precisa.

La ausencia de actitud propositiva del gerente, invalida o menoscaba sus niveles de conocimientos y habilidades adquiridas (“... sabe y puede, pero no quiere...").

\section{REFERENCIAS BIBLIOGRÁFICAS}

Alvarez, de Sayas. (2012) La Teoría de la Gestión desde la complejidad. Bolivia. La Paz Bolivia: Kipu

Blutman y Felcman, G. I. (2011). Nuevos modelos de gestión pública. Buenos Aires Argentina: Temas.

Chesman, Y. A. (2010). Propuesta de Política Nacional de Modernización de la Gestión Pública al 2021. Lima.: GTZ. P. 3.

Chiavenato, I. (2011). Gestión del talento humano. México: Mac Graw Hill

Duque, F. (2006). El Gerente público un profesional indispensable para el desarrollo. Cuaderno EBAPE. BR., 1 - 2

Galvez, G. L. (2016). Gestión del Talento Humano en el sector público de Colombia. Apuntes de Administración, 21.

Longo, F. (2002). El Desarrollo de competencias directivas en los sistemas públicos. Madrid - España:ESADE.CLAD.P.8.

Vallejos, L.M (2015) Gestión del Talento Humano. Ecuador: La Caracola.

Castillo Aponte, J. (17 de febrero de 2018). Gestión del Talento Humano. Obtenido de reforma 355: http:/www.crecimiento integral.net/

Michaels, Handfield and Beth (2003). La Guerra por el Talento. Madrid - España. Club de Gestión.

Pagani, M.L. (12 de agosto de 2015). Estudios sobre gestión pública. Obtenido de www. Modernización.gba.gob.ar/expertos/:http//www.modernización.gba.gob.ar.com

Gálvez, G.L. Gestión del Talento Humano en el sector público de Colombia. Apuntes de administración,21.

Carta iberoamericana de la Función pública. CLAD, (2003). XXIII Cumbre iberoamericana, Bolivia 